\section{Feasibility and efficacy of addition of individualized-dose lenalidomide to chlorambucil and rituximab as first-line treatment in elderly and FCR-unfit patients with advanced chronic lymphocytic leukemia}

\author{
Arnon P. Kater, ${ }^{1}$ Marinus H.J. van Oers, ${ }^{1}$ Yvette van Norden, ${ }^{2}$ Lina van der \\ Straten, ${ }^{3}$ Julia Driessen, ${ }^{1}$ Ward F.M. Posthuma, ${ }^{4,5}$ Martin Schipperus, ${ }^{6}$ Martine \\ E.D. Chamuleau, ${ }^{7}$ Marcel Nijland, ${ }^{8}$ Jeanette K. Doorduijn, ${ }^{9}$ Michel Van Gelder, ${ }^{10}$ \\ Mels Hoogendoorn, ${ }^{11}$ Francien De Croon, ${ }^{12}$ Shulamiet Wittebol, ${ }^{13} \mathrm{~J}$. Martijn \\ Kerst, ${ }^{14}$ Erik W.A. Marijt, ${ }^{5}$ Reinier A.P. Raymakers, ${ }^{15}$ Martijn R. Schaafsma, ${ }^{16}$ \\ Johan A. Dobber, ${ }^{17}$ Sabina Kersting ${ }^{6}$ and Mark-David Levin ${ }^{3}$ on behalf of the \\ HOVON CLL study group
}

\begin{abstract}
${ }^{1}$ Department of Hematology and Lymphoma and Myeloma Center Amsterdam, Academic Medical Center, Amsterdam; ${ }^{2}$ Department of Hematology - HOVON Data Center, Erasmus MC Cancer Institute, Rotterdam; ${ }^{3}$ Department of Internal Medicine, Albert Schweitzer Hospital, Dordrecht; ${ }^{4}$ Department of Internal Medicine, Reinier de Graaf Hospital, Delft; ${ }^{5}$ Department of Hematology, Leiden University Medical Center; ' ${ }^{6}$ epartment of Hematology, Haga Hospital, the Hague; ' Department of Hematology, VU University Medical Center, Amsterdam; ${ }^{8}$ Department of Hematology, University Medical Center, Groningen; ${ }^{9}$ Department of Hematology, Erasmus MC Cancer Institute, Rotterdam; ${ }^{10}$ Department of Hematology, Maastricht University Medical Center; ${ }^{11}$ Department of Internal Medicine, Medical Center, Leeuwarden; ${ }^{12}$ Department of Internal Medicine, Ikazia Hospital, Rotterdam; ${ }^{13}$ Department of Internal Medicine, Gelderland Valley Hospital, Ede; ${ }^{14}$ Department of Medical Oncology, Antoni van Leeuwenhoek Hospital, Amsterdam; ${ }^{15}$ Department of Hematology, University Medical Center, Utrecht; ${ }^{16}$ Department of Hematology, Medical Spectrum Twente, Enschede and ${ }^{17}$ Laboratory Special Hematology, Academic Medical Center, Amsterdam, the Netherlands
\end{abstract}

\section{ABSTRACT}

L enalidomide has been proven to be effective but with a distinct and difficult to manage toxicity profile in the context of chronic lymphocytic leukemia, potentially hampering combination treatment with this drug. We conducted a phase 1-2 study to evaluate the efficacy and safety of six cycles of chlorambucil $\left(7 \mathrm{mg} / \mathrm{m}^{2}\right.$ daily), rituximab (375 $\mathrm{mg} / \mathrm{m}^{2}$ cycle 1 and $500 \mathrm{mg} / \mathrm{m}^{2}$ cycles 2-6) and individually-dosed lenalidomide (escalated from $2.5 \mathrm{mg}$ to $10 \mathrm{mg}$ ) (induction-I) in first-line treatment of patients with chronic lymphocytic leukemia unfit for treatment with fludarabine, cyclophosphamide and rituximab. This was followed by 6 months of $10 \mathrm{mg}$ lenalidomide monotherapy (induction-II). Of 53 evaluable patients in phase 2 of the study, 47 (89\%) completed induction-I and $36(68 \%)$ completed induction-II. In an intention-to-treat analysis, the overall response rate was $83 \%$. The median progressionfree survival was 49 months, after a median follow-up time of 27 months. The 2- and 3-year progression-free survival rates were $58 \%$ and $54 \%$, respectively. The corresponding rates for overall survival were $98 \%$ and $95 \%$. No tumor lysis syndrome was observed, while tumor flair reaction occurred in five patients (9\%, 1 grade 3$)$. The most common hematologic toxicity was grade 3-4 neutropenia, which occurred in $73 \%$ of the patients. In conclusion, addition of lenalidomide to a chemotherapy backbone followed by a fixed duration of lenalidomide monotherapy resulted in high remission rates and progression-free survival rates, which seem comparable to those observed with novel drug combinations including novel CD20 monoclonal antibodies or kinase inhibitors. Although lenalidomide-specific toxicity remains a concern, an individualized dose-escalation schedule is feasible and results in an acceptable toxicity profile. EuraCT number: 2010-022294-34.
Haematologica 2019

Volume 104(1):147-154

\section{Correspondence:}

a.p.kater@amc.uva.nl

Received: March 28, 2018.

Accepted: August 9, 2018.

Pre-published: August 16, 2018.

doi:10.3324/haematol.2018.193854

Check the online version for the most updated information on this article, online supplements, and information on authorship \& disclosures: www.haematologica.org/content/104/1/147

\section{(C)2019 Ferrata Storti Foundation}

Material published in Haematologica is covered by copyright. All rights are reserved to the Ferrata Storti Foundation. Use of published material is allowed under the following terms and conditions:

https://creativecommons.org/licenses/by-nc/4.0/legalcode. Copies of published material are allowed for personal or internal use. Sharing published material for non-commercial purposes is subject to the following conditions:

https://creativecommons.org/licenses/by-nc/4.0/legalcode, sect. 3. Reproducing and sharing published material for commercial purposes is not allowed without permission in writing from the publisher. 


\section{Introduction}

Although important progress has been made in the management of chronic lymphocytic leukemia (CLL) in the last decades, treatment for elderly and unfit patients is still not optimized. Despite clear advantages of the chemotherapeutic drug chlorambucil in elderly patients, with respect to toxicity, oral administration and low costs, its efficacy as a single agent is low in CLL.

The CLL11 trial found that the progression-free survival of patients given combination treatment with chlorambucil and rituximab or chlorambucil and obinutuzumab was longer than that of patients given chlorambucil monotherapy (median progression-free survival: 15.4 months and 29.2 months, respectively, versus 11.1 months). ${ }^{1}$ Since the publication of the findings of this trial, treatment with chlorambucil and an anti-CD20 monoclonal antibody has become the standard, first-line therapy for elderly patients and those unfit for treatment with fludarabine, cyclophosphamide and rituximab (FCR). ${ }^{2}$ Nevertheless, relapses occur in virtually all patients within 3.5 years. $^{3}$ A more recent trial compared chlorambucil monotherapy with continuous treatment with the Bruton tyrosine kinase inhibitor ibrutinib and showed improved progression-free survival and overall survival in patients treated in the ibrutinib arm. ${ }^{4}$ Based on this study, ibrutinib also acquired a label for first-line treatment for previously untreated FCR-unfit patients. However, treatment with targeted inhibitors is not considered curative, most likely because of the pronounced evolutionary capacity CLL cells resulting in the emergence of drug-resistant clones. ${ }^{5}$ Moreover, prolonged treatment with targeted inhibitors has significant medical, social, and economic costs. It is, therefore, necessary to optimize therapy for elderly and FCR-unfit patients and combine therapies with other mechanisms of action.

Lenalidomide is an oral immunomodulatory drug that has multiple mechanisms of action on the immune system. It alters the interaction between CLL cells and the protective microenvironment and stimulates the cytotoxicity of natural killer cells against CLL cells. Lenalidomide restores the immunological synapse between T cells and CLL cells, reversing $\mathrm{T}$-cell dysfunction and enhancing the ability of immune recognition of tumor cells. In addition, lenalidomide directly affects cell proliferation through upregulation of p21 activity. This effect is independent of the TP53 pathway and could thus be applicable for patients with TP53 dysfunction too. ${ }^{6-8}$

The overall response rates to lenalidomide as a single agent are low with few complete remissions (overall response rate range: $32 \%-72 \%$, complete remission rate range: $0 \%-10 \%$ ) in previously untreated or treated CLL patients. ${ }^{9-13}$ Addition of rituximab to lenalidomide resulted in an increased overall response rate of $78 \%$ with complete remissions in $11 \%$ of patients with relapsed/refractory CLL. ${ }^{14-16}$ Moreover, addition of rituximab seems to diminish tumor lysis syndrome and tumor flare reaction, which are distinct and difficult to manage toxicity profiles reported in CLL patients treated with lenalidomide. ${ }^{16}$

Given the key role of the microenvironment in chemoresistance, addition of lenalidomide to chlorambucil and rituximab may result in further improvement of response rates. ${ }^{6}$ Furthermore, extended duration of treatment has been reported to improve both the overall response rate and quality of responses. ${ }^{16}$

Currently it is not known whether combination treatment with lenalidomide, rituximab and chlorambucil is feasible in terms of safety and efficacy. As such, we conducted a phase 1-2 study in which six cycles of triple therapy followed by six cycles of lenalidomide monotherapy were tested in elderly and FCR-unfit patients with advanced, previously untreated CLL.

\section{Methods}

\section{Study design and patients}

This prospective, open-label study consisted of a phase 1 dose-finding part and a phase 2 efficacy part. Treatment-naïve patients diagnosed with immunophenotypically confirmed CLL, aged 65 - 80 years or 18 - 64 years with a Cumulative Illness Rating Scale score $\geq 7$, in Binet C (Rai III-IV) stage, or with confirmed active disease in Binet A or B (Rai 0-II) stage were enrolled. ${ }^{17}$ A complete list of inclusion and exclusion criteria is presented in Online Supplementary Table S1.

\section{Treatment}

All patients were treated with six cycles (every 28 days) of a combination of chlorambucil (p.o. cycles 1-6, days $1-7$ ), ritux-
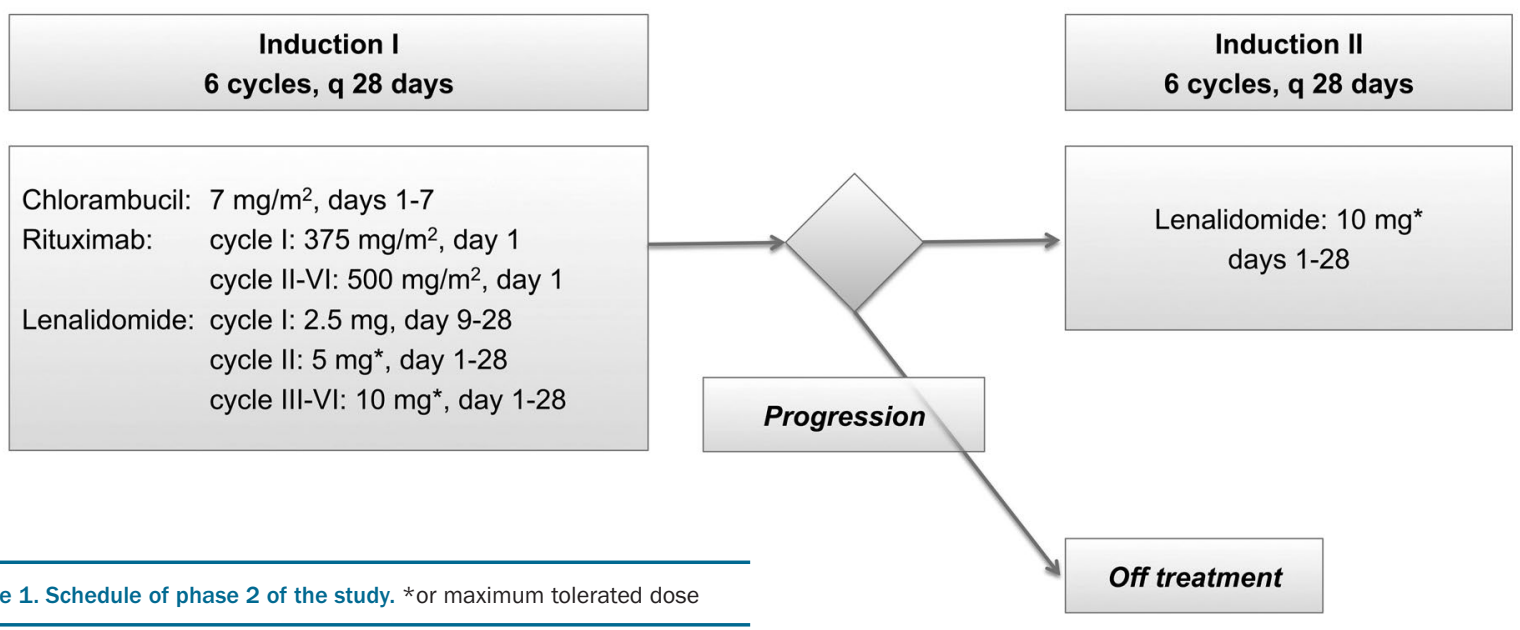
imab $\left(375 \mathrm{mg} / \mathrm{m}^{2}\right.$ i.v. cycle 1 , day $1 ; 500 \mathrm{mg} / \mathrm{m}^{2}$ i.v. cycles $2-6$, day 1) and lenalidomide (induction-I). The dose of lenalidomide was escalated from $2.5 \mathrm{mg}$ to $10 \mathrm{mg}$ during induction-I. Subsequently patients were treated with six cycles of lenalidomide $10 \mathrm{mg}$ p.o. daily (induction-II) (Figure 1). The criteria for discontinuation and restarting lenalidomide and prophylactic treatment are presented in Online Supplementary Tables S2, S3, and $S 4$, respectively.

\section{Study phase 1}

This phase of the study focused on determining the maximum tolerated dose and the recommended dose level of chlorambucil in combination with rituximab and lenalidomide. Six patients started combination treatment with chlorambucil at dose level 1 $(7 \mathrm{mg})$. If no more than one dose-limiting toxicity occurred (Online Supplementary Table S5), the dose was escalated to $10 \mathrm{mg}$ (dose level 2) for the subsequent patients, on the basis of which the recommended dose level was established.

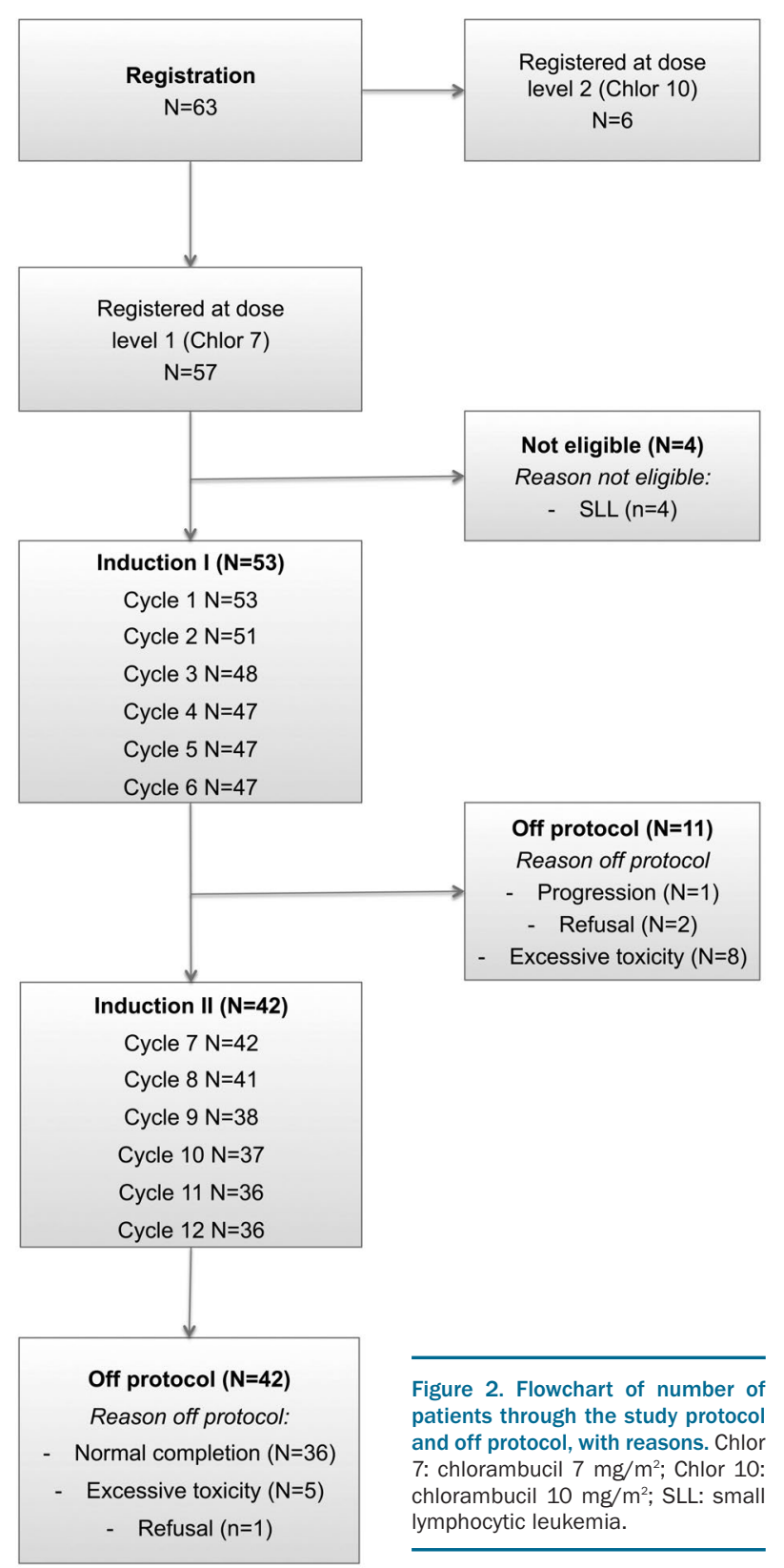

\section{Study phase 2}

The aim of this phase was to evaluate the efficacy of chlorambucil, at the recommended dose level, in combination with rituximab and lenalidomide, in terms of overall response rate.

\section{Endpoints}

The protocol endpoints are presented in Online Supplementary Table S6. Responses were determined according to the 2008 International Workshop on CLL criteria ${ }^{17}$ and evaluated after cycle 3 (clinically), after cycle 6 , at the end of the study treatment and during follow-up. The presence of minimal residual disease, as measured in the peripheral blood, was determined centrally using six-color flow cytometry. ${ }^{18}$ Toxicity was reported according to the National Cancer Institute Common Terminology Criteria for Adverse Events (CTCAE) version 4.0. The Cairo-Bishop grading classification ${ }^{19}$ and CTCAE version $3.0^{20}$ were used to grade tumor lysis syndrome and tumor flare reaction, respectively. ${ }^{20}$

\section{Statistical analysis}

All analyses were performed according to the intention-totreat principle but, in agreement with the protocol, excluding patients not considered eligible in hindsight. The patients' characteristics and treatment toxicity were summarized by descriptive cross-tabulations. Responses were tabulated according to the fraction of the optimal dose of lenalidomide in each cycle $(<90 \%$ versus $\geq 90 \%)$. The Kaplan-Meier method was used for time-to-event analysis. All statistical analyses were performed using STATA Statistical Software version 14.

\section{Ethics}

Written informed consent was obtained before enrollment in the trial. The study was approved by an accredited Ethical Committee and Institutional Review Board and was performed according to the Declaration of Helsinki, the International Conference on Harmonization Good Clinical Practice Guidelines and the European Union Clinical Trial Directive (2001/20/EG). The study was registered with EuraCT number 2010-022294-34.

\section{Results}

Between September 20, 2011 and October 18, 2015, 63 previously untreated patients with CLL from 26 centers in the Netherlands were enrolled in this study.

\section{Maximum tolerated dose and recommended dose level of chlorambucil given in combination with rituximab and lenalidomide}

Twelve patients were included in phase 1 of this study aimed at determining the recommended dose level of chlorambucil in combination with rituximab and lenalidomide. In the first dose level group $7 \mathrm{mg} / \mathrm{m}^{2}$ chlorambucil), no dose-limiting toxicity was observed. Subsequently, six patients were included in the second dose level, of $10 \mathrm{mg} / \mathrm{m}^{2}$, of chlorambucil. Again, no doselimiting toxicities were observed at this dose level. There were no differences in the proportions of adverse or severe adverse events between the groups treated with the two different doses. Lenalidomide dose modifications were more frequently applied in the second dose level group. Despite the lack of significant differences in toxicity between the two groups treated with different chlorambucil dosages, the principal investigators, with sup- 
port of the data safety and monitoring board, decided to continue part 2 of the trial with the recommended dose level of $7 \mathrm{mg} / \mathrm{m}^{2}$, day $1-7$, of chlorambucil (dose level 1) based on toxicity reports of an international phase 3 study. ${ }^{9}$

\section{Phase 2 patients and study treatment}

For the phase 2 part of the trial, 57 patients were included of whom four were subsequently excluded because they were not eligible in hindsight having been found to have small lymphocytic lymphoma. Combination treatment with chlorambucil, rituximab and lenalidomide was started in 53 patients (induction-I). The patients' disposition through the trial is summarized in Figure 2 and the clinical, biological and cytogenetic characteristics of the patients are reported in Table 1. The median age of the patients was 71 years (range, $60-80$ ). Mutational status could be assessed in 39 patients. The IGVH genes were mutated in 20 patients (51\%) and unmutated in 19 patients $(49 \%)$. Deletion of chromosome $17 p$ was found in eight of $51(17 \%)$ patients. Eight of $50(15 \%)$ and 23 of $50(43 \%)$ patients had deletion of chromosome 11q and 13q, respectively.

Forty-seven $(89 \%)$ patients completed the six planned courses of chlorambucil, rituximab and lenalidomide. Treatment was prematurely discontinued in 11 patients (21\%). Reasons for discontinuation were excessive toxic-

Table 1. Patients' characteristics.

\begin{tabular}{|c|c|}
\hline Patients' characteristics & umber of patients $(n=53)$ \\
\hline $\begin{array}{l}\text { Median age [range], years } \\
\text { Age, } \mathrm{n} .(\%) \\
<65 \text { years } \\
\geq 65 \text { to } \leq 70 \text { years } \\
>70 \text { years }\end{array}$ & $\begin{array}{c}71[60-80] \\
1(2) \\
25(47) \\
27(51)\end{array}$ \\
\hline Male sex, n. (\%) & $29(55)$ \\
\hline $\begin{array}{l}\text { RAI stage, n. (\%) } \\
0-\mathrm{I} \\
\text { II } \\
\text { III } \\
\text { IV }\end{array}$ & $\begin{array}{l}11(21) \\
10(19) \\
22(42) \\
10(19)\end{array}$ \\
\hline $\begin{array}{l}\text { CIRS score }-\mathrm{n} .(\%) \\
\leq 6 \\
>6 \\
\text { Unknown }\end{array}$ & $\begin{array}{l}47(87) \\
4(8) \\
2(4)\end{array}$ \\
\hline Median CIRS [range] & $1[0-9]$ \\
\hline $\begin{array}{l}\text { Lactate dehydrogenase, } \mathrm{n} .(\%) \\
\text { Lactate dehydrogenase } \leq \text { upper level of norma } \\
\text { Lactate dehydrogenase }>\text { upper level of norma } \\
\text { Unknown }\end{array}$ & $\begin{array}{l}33(62) \\
18(34) \\
2(4)\end{array}$ \\
\hline Median $\beta 2$-microglobulin [range] $(\mathrm{n}=43)$ & $3.8[1.6-10.4]$ \\
\hline $\begin{array}{l}\text { IGVH mutational status }-\mathrm{n} .(\%) \\
\text { Mutated } \\
\text { Unmutated } \\
\text { Unknown }\end{array}$ & $\begin{array}{l}20(38) \\
19(36) \\
14(26)\end{array}$ \\
\hline $\begin{array}{l}\text { Cytogenetic abnormalities - n. (\%) } \\
\text { Dell7p } \\
\text { Dell1q } \\
\text { Trisomy } 12 \\
\text { Del 13q }\end{array}$ & $\begin{array}{l}8 \text { out of } 51(17 \%) \\
8 \text { out of } 51(17 \%) \\
12 \text { out of } 50(24 \%) \\
23 \text { out of } 50(46 \%)\end{array}$ \\
\hline
\end{tabular}

$\overline{\text { CRS: Cumulative Illness Rating Scale; IGVH: immunoglobulin variable region heavy }}$ chain; del: deletion. ity ( $n=8: 4$ cases of skin toxicity, 1 grade 3 allergic reaction, 1 case of neuropathy, 1 acute coronary syndrome and 1 case of mucositis), refusal $(n=2)$ and progression $(n=1)$.

Following combination treatment, lenalidomide monotherapy was initiated in 42 patients $(79 \%)$ (induction-II). Treatment was prematurely discontinued during induction-II in six patients, due to excessive toxicity $(\mathrm{n}=5$ : 3 cases of hematologic toxicity in the form of persistent neutropenia and 2 cases of diarrhea with no improvement following dose reductions.) or refusal $(n=1)$. Treatment was completed according to the protocol in 36 (68\%) patients.

The full dose of lenalidomide during induction-I was given to $76 \%$ of the patients in cycle 1 , to $57 \%$ of the patients in cycle 2 , to $50 \%$ in cycle $3,57 \%$ in cycle $4,53 \%$ in cycle 5 and $51 \%$ in cycle 6 (Figure 3). During cycle 6 of induction-II, $25(69 \%)$ patients received lenalidomide at the full dose. The median dose of lenalidomide according to the prescribed protocol dosing was $86.7 \%$ (range, $10 \%-101 \%$ ) and $99.7 \%$ (range, $25 \%-104 \%$ ) in inductionI and induction-II, respectively.

\section{Response evaluation}

On an intention-to-treat basis, response was analyzed in 53 patients at the end of induction-I. The overall response rate was $83 \%$ (95\% confidence interval: $72 \%$ $92 \%$ ), which resulted in a positive trial based on the phase 2 design as stated in the protocol. The responding patients all achieved a partial response and no complete responses were observed on induction-I (Figure 4).

Responses at the end of induction-II were evaluated in all patients $(n=42)$ who started this phase of induction. The overall response rate was $93 \%$ (95\% confidence interval: $79 \%-98 \%)$ and consisted of $14 \%$ complete responses $(n=6)$ and $79 \%$ partial responses $(n=33)$. The disease remained stable in two $(5 \%)$ patients and pro-

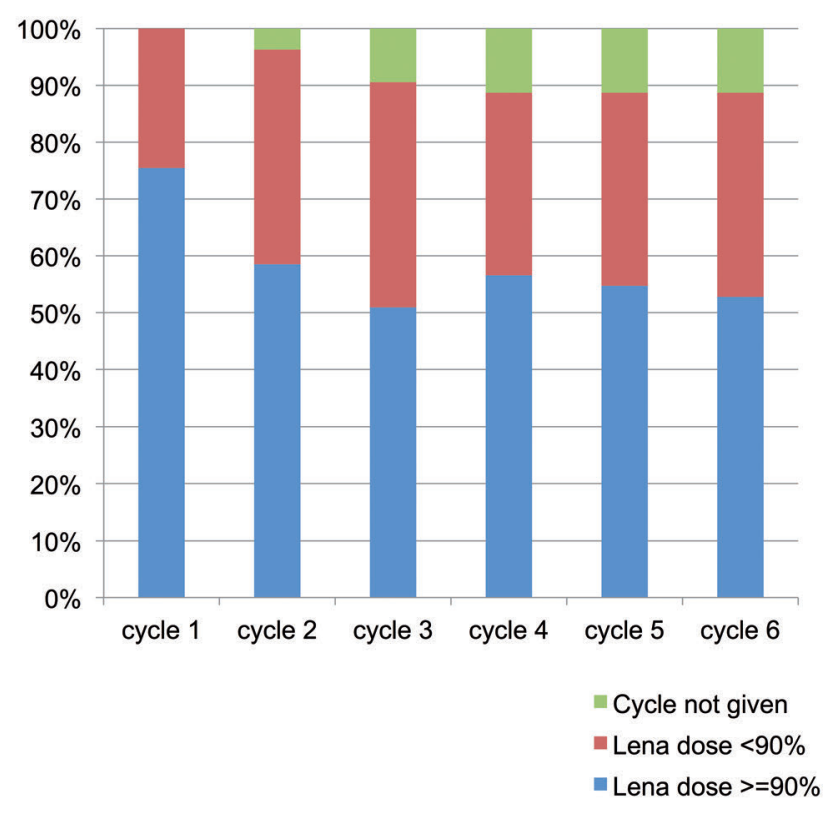

Figure 3. Lenalidomide dosing: cumulative dose of lenalidomide compared to optimal dose per treatment cycle during induction-l. Lena: Lenalidomide. 
gressed in one (2\%) patient. Improvement of the response after induction-II was observed in eight $(15 \%)$ of all patients who started induction-I: six patients $(11 \%)$ had an improvement from a partial to complete response and two patients (4\%) had an improvement from stable disease to a partial response in induction-II.

Flow-based minimal residual disease analysis was performed on peripheral blood in 41 patients. Of these patients, four $(8 \%)$ achieved minimal residual disease negativity after induction-I and an additional two (4\%) after induction-II.

\section{Survival}

After a median follow-up of 27 months, the median progression-free survival was 49 months (Figure 5A). At 2 and 3 years, $58 \%$ [standard error $(\mathrm{SE})=8 \%$ ] and $54 \%(\mathrm{SE}=8 \%)$ of the patients, respectively, were alive without progression. The 3-year progression-free survival rate of patients with a deletion of chromosome $17 p$ [del(17p); $n=8]$ was lower than that of patients without this deletion (38\% versus $59 \%$, respectively). For patients who started inductionII $(n=42)$, the subsequent progression-free survival was 41 months and the 2-year progression-free survival rate was $56 \%$ (SE=9\%). The median event-free survival was 49 months and the event-free survival rate at 3 years was $53 \%$ $(\mathrm{SE}=8 \%$ ) with $13 \%(\mathrm{SE}=5 \%$ ) non-responders and $34 \%$ $(\mathrm{SE}=8 \%)$ with progressive disease. Of the patients with progressive disease, one patient started the next treatment before progressing. During follow-up the median overall survival was not reached with 2- and 3-year overall survival rates of $98 \%(\mathrm{SE}=2 \%)$ and $95 \%(\mathrm{SE}=3 \%)$, respectively (Figure $5 \mathrm{~B}$ ). With regard to overall survival following induction-II, no deaths had occurred among the patients who started induction-II.

\section{Safety}

Two patients included in the phase 2 part of the trial developed a grade 4 adverse event, consisting of neutropenic sepsis. These severe adverse events occurred during induction-I in cycle 2 and cycle 5 . No grade 4 adverse events were observed during induction-II. Tumor lysis syndrome did not occur. A tumor flare reaction was reported in five patients $(9 \%)$ and was s grade 2 in four patients and grade 3 in one patient. Six patients developed a secondary malignancy, which was localized skin cancer in all but one. One patient had a solid tumor.

Grade 3-4 neutropenia occurred in $39(73 \%)$ and 27 $(64 \%)$ patients during induction-I and induction-II, respectively, which prompted granulocyte colony-stimulating factor administration as shown per cycle in Online

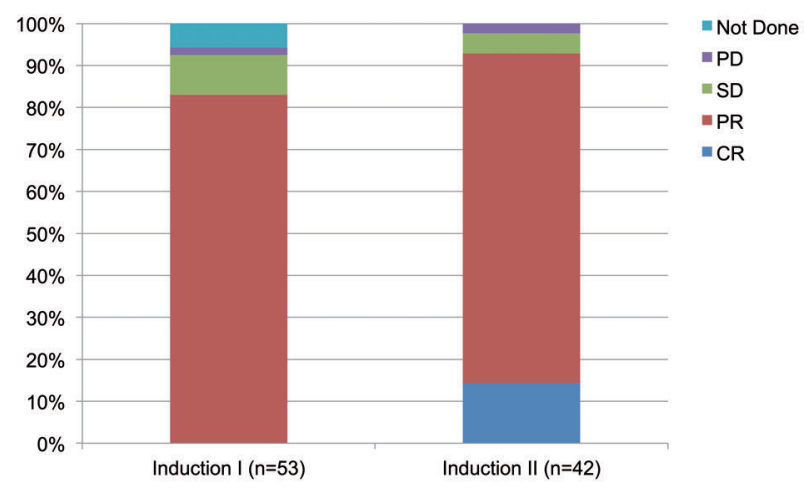

Figure 4. Responses after induction-I and -II. PD: progressive disease; SD: stable disease; PR: partial response; CR: complete response.

Table 2. Grade 3-4 toxicities.

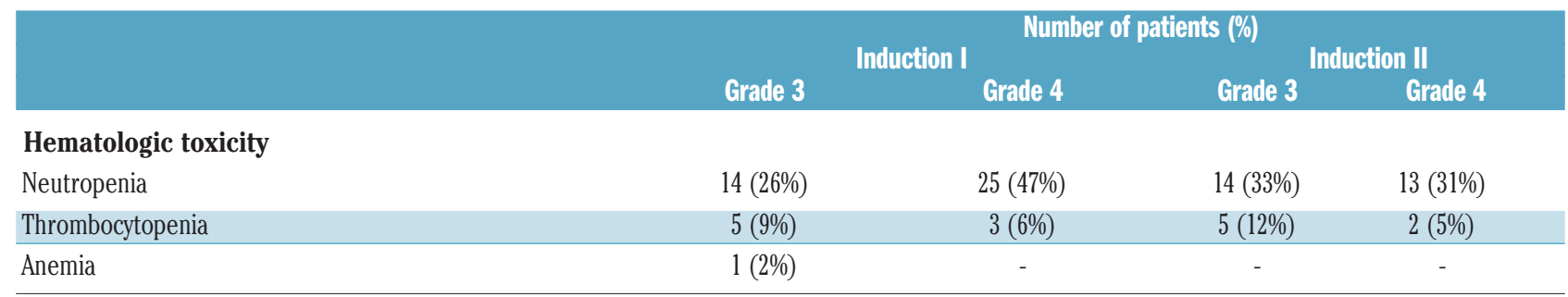

\section{Other adverse events}

\begin{tabular}{|c|c|c|c|c|}
\hline Any & $21(40 \%)$ & $2(4 \%)$ & $8(19 \%)$ & - \\
\hline Infections and infestations & $5(9 \%)$ & - & $4(10 \%)$ & - \\
\hline General disorders and administration site conditions & $6(11 \%)$ & - & - & - \\
\hline Skin and subcutaneous tissue disorders & $6(11 \%)$ & - & $3(7 \%)$ & - \\
\hline Respiratory, thoracic and mediastinal disorders & $3(6 \%)$ & - & - & - \\
\hline Musculoskeletal and connective tissue disorders & $1(2 \%)$ & - & - & - \\
\hline Nervous system disorders & $1(2 \%)$ & - & - & - \\
\hline Blood and lymphatic disorders & $1(2 \%)$ & - & - & - \\
\hline Cardiac disorders & $1(2 \%)$ & $1(2 \%)$ & $1(2 \%)$ & - \\
\hline Immune system disorders & $2(4 \%)$ & - & - & - \\
\hline Metabolism and nutrition disorders & $2(4 \%)$ & - & - & - \\
\hline Renal and urinary disorders & $1(2 \%)$ & - & - & - \\
\hline Investigations & $1(2 \%)$ & $1(2 \%)$ & - & - \\
\hline Psychiatric disorders & $1(2 \%)$ & - & - & - \\
\hline
\end{tabular}


A

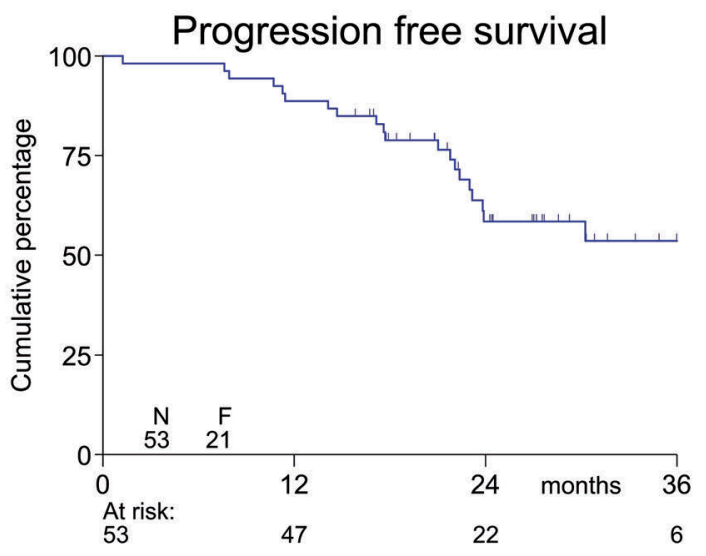

B

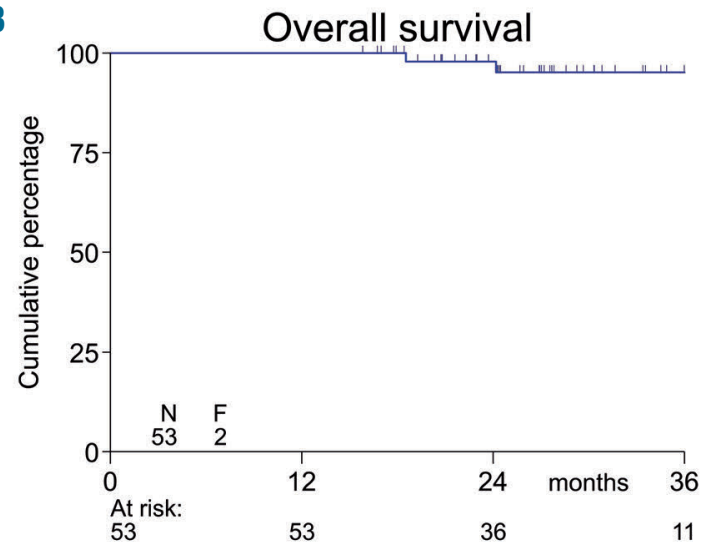

Figure 5. Survival outcomes after registration. (A) Progression-free survival and (B) overall survival, with the numbers of patients at risk at 1,2 and 3 years.

Supplementary Table S7. Neutropenic sepsis occurred in two (4\%) patients. Grade 3-4 thrombocytopenia was recorded in $15 \%$ and $17 \%$ of the patients after inductionI and induction-II, respectively. Despite this, no grade 3-4 bleeds occurred. Grade 3-4 anemia was recorded in 2\% of the patients during induction-I. Although all patients received thromboembolic prophylaxis, two patients (4\%) had a thromboembolic event (i.e. deep vein thrombosis and thrombophlebitis despite prophylactic aspirin).

Grade 3-4 skin and subcutaneous tissue disorders occurred in six patients $(11 \%)$. Thirteen patients $(25 \%)$ developed grade 2 skin toxicity. Other grade 3-4 toxicities occurred in 26 patients (49\%), of which $23(44 \%)$ during induction-I and eight (19\%) during induction-II. The majority of these toxic events were infections (15\%), gastrointestinal disorders $(15 \%)$, or general disorders and administration site conditions (11\%) (Table 2).

Two patients $(4 \%)$ have died during disease progression. These deaths occurred 19 and 24 months after registration in the study.

\section{Discussion}

In this prospective, open label, phase $1 / 2$ study, we investigated the activity of lenalidomide in combination with chlorambucil and rituximab for elderly and FCRunfit patients with previously untreated CLL.

After a median follow-up of 27 months, the median progression-free survival in our study was 49 months. The 2- and 3-year progression-free survival rates were $58 \%$ and $54 \%$, respectively. The corresponding overall survival rates were $98 \%$ and $95 \%$. Since this study is the first to investigate triple treatment with chlorambucil, rituximab and lenalidomide, direct comparison with other clinical trials is limited.

Recently, novel first-line regimens have been tested in frail, elderly patients: e.g., chlorambucil as a backbone with novel CD20 monoclonal antibodies and novel chemotherapy-free combinations. The CLL11 trial compared treatment with chlorambucil monotherapy, chlorambucil and rituximab or chlorambucil and obinutuzumab. ${ }^{1}$ The median progression-free survival was 16.3 months for patients treated with chlorambucil and rituximab and 26.7 months for those treated with chlorambucil and obinutuzumab. The COMPLEMENT-1 trial investigated treatment with chlorambucil and ofatumumab, comparing this with chlorambucil monotherapy. ${ }^{21}$ After a median follow-up of 29 months, the median progression-free survival was 22 months and the overall response rate was $82 \%$ for patients treated with chlorambucil and ofatumumab.

With regards to chemotherapy-free combinations of lenalidomide with rituximab, an overall response rate of $88 \%$ and a median progression-free survival of 20 months were observed in a phase 2 study. ${ }^{14}$ In the phase $1 \mathrm{~b} / 2$ PCYC-1102/1103 studies, treatment with ibrutinib resulted in overall response rates of $87 \%$ and $89 \%$ in treatment-naïve and relapsed/refractory patients, respectively. The overall response rates were $97 \%, 89 \%$ and $79 \%$ in relapsed/refractor patients with a del(11q), complex karyotype and del(17p), respectively. After a median followup of 61.5 months, the median progression-free survival had not been reached in treatment-naïve patients and was 51 months in relapsed/refractory patients. 2,23

With all the caveats necessary when comparing different trials, the progression-free survival and overall response rates in the current study seem at least comparable to those observed with chlorambucil in combination with novel CD20 monoclonal antibodies and those observed with kinase inhibitors.

The dose of chlorambucil used in our study was $7 \mathrm{mg} / \mathrm{m}^{2}$ on days 1-7 of cycles 1-6. Chlorambucil was administered in combination with rituximab and lenalidomide during six cycles. In the COMPLEMENT-1 trial patients were treated with $10 \mathrm{mg} / \mathrm{m}^{2}$ of chlorambucil on days $1-7$ during a minimum of three and a maximum of 12 cycles. In the CLL11 trial, chlorambucil was administered at a dose of 0.5 $\mathrm{mg} / \mathrm{kg}$ on days 1 and 15 of cycles $1-6$.

Lenalidomide was started at a dose of $2.5 \mathrm{mg} /$ day, and was steadily increased to $10 \mathrm{mg} /$ day which was maintained until cycle 6 , provided there were no dose-limiting toxicities. The escalation scheme of lenalidomide was previously described in combination with rituximab in a phase 2 trial. ${ }^{14}$ However, in that study lenalidomide was administered for 21 days, followed by a period of rest each cycle. The use of a continuous dose of lenalidomide 
in combination with rituximab was previously described and was proven to be safe and effective. ${ }^{15,24}$

Following induction-I, patients received an additional six cycles of monotherapy with lenalidomide $10 \mathrm{mg}$ /day. As previously reported, responses can improve with lenalidomide consolidation treatment. ${ }^{11,25-27}$ In addition, the CLLM1 study showed that lenalidomide (5-10-15 $\mathrm{mg} /$ day) can be efficaciously used as maintenance treatment, prolonging the time to progression as compared with placebo, in first-line patients with CLL who do not achieve minimal residual disease negativity following chemoimmunotherapy induction. ${ }^{25}$

Although lenalidomide-specific toxicity remains a concern, an individualized dose-escalation schedule is feasible and results in an acceptable toxicity profile and less frequent occurrence of tumor lysis syndrome and tumor flare reactions. Grade 3-4 toxicities were reported in 26 (49\%) patients. The most frequently reported toxicities were infections and gastrointestinal disorders. Despite prophylaxis with granulocyte colony-stimulating factor, $64 \%$ and $73 \%$ of patients developed grade $3-4$ neutropenia. Similar percentages were observed in the GIMEMA trial, in which patients were treated with a combination of fludarabine, lenalidomide and rituximab. ${ }^{9}$ The rate of grade 3-4 toxicities reported with ofatumumab and chlorambucil in the COMPLEMENT-1 trial was $50 \%,{ }^{21}$ which is comparable to the rate in our study. Treatment with ibrutinib, as described in the PCYC-1102/1103 studies, ${ }^{4}$ was less toxic than treatment with chlorambucil, rituximab and lenalidomide.

The ORIGIN study, in which lenalidomide monotherapy was compared with chlorambucil, was stopped prematurely after an imbalance in deaths and treatment- emergent adverse events in the lenalidomide arm. ${ }^{10}$ Based on these observations, lenalidomide monotherapy was not recommended as first-line treatment in CLL patients, particularly in those who are elderly and/or frail. Although our study has shown that adverse events and deaths can be reduced by using individualized dose schedules, intensive monitoring is required. The high death rate observed in the ORIGIN study in lenalidomide-treated patients ${ }^{10}$ was not replicated in our study.

Due to rapid developments, the clinical impact of our study might be limited. Although chemo-immunotherapy is still considered a standard first-line option in CLL patients without del17p/TP53 mutation, phase 2 as well as phase 3 studies examining new chemotherapy-free regimens show a high proportion of minimal residual disease-negative responses. ${ }^{28}$ Nevertheless, data currently available imply that patients will relapse even following treatment with novel agents. Based on the findings of this study and on lenalidomide's unique mechanisms of action, ${ }^{6}$ there might be a role for this drug or for the newer immunomodulatory treatments either in combination with novel agents, or in patients who are not eligible for novel therapies such as ibrutinib.

In conclusion, our study showed that triple treatment with chlorambucil, rituximab and lenalidomide is an effective therapy in previously untreated elderly and FCR-unfit patients with CLL. Intensive monitoring is of paramount importance to minimize toxicity and ensure safety.

\section{Acknowledgments}

The authors would like to thank the HOVON109-trial team of the Hovon Data Centre for their help with the trial management and central data management.

\section{References}

1. Goede V, Fischer K, Busch R, et al. Obinutuzumab plus chlorambucil in patients with CLL and coexisting conditions. N Eng J Med. 2014;370(12):11011110.

2. Hallek M. Chronic lymphocytic leukemia: 2017 update on diagnosis, risk stratification, and treatment. Am J Hematol. 2017;92(9):946-965.

3. Goede V, Fischer K, Engelke A, et al. Obinutuzumab as frontline treatment of chronic lymphocytic leukemia: updated results of the CLL11 study. Leukemia. 2015;29:16024.

4. Burger JA, Tedeschi A, Barr PM, et al. Ibrutinib as initial therapy for patients with chronic lymphocytic leukemia. N Eng J Med. 2015;373(25):2425-2437.

5. Landau DA, Sun C, Rosebrock D, et al. The evolutionary landscape of chronic lymphocytic leukemia treated with ibrutinib targeted therapy. Nat Commun. 2017;8(1): 2185.

6. Kater AP, Tonino SH, Egle A, Ramsay AG. How does lenalidomide target the chronic lymphocytic leukemia microenvironment? Blood. 2014;124(14):2184-2189.

7. Itchaki G, Brown JR. Lenalidomide in the treatment of chronic lymphocytic leukemia. Expert Opin Investig Drugs. 2017;26(5):633-650.

8. Browning RL, Byrd WH, Gupta N, et al. Lenalidomide induces interleukin-21 production by $T$ cells and enhances IL21-mediated cytotoxicity in chronic lymphocytic leukemia B cells. Cancer Immunol Res. 2016;4(8):698-707.

9. Mauro FR, Carella AM, Molica S, et al. Fludarabine, cyclophosphamide and lenalidomide in patients with relapsed/refractory chronic lymphocytic leukemia. A multicenter phase I-II GIMEMA trial. Leuk Lymphoma. 2017;58(7):1640-1647.

10. Chanan-Khan A, Egyed M, Robak T, et al. Randomized phase 3 study of lenalidomide versus chlorambucil as first-line therapy for older patients with chronic lymphocytic leukemia (the ORIGIN trial). Leukemia. 2017;31(5):1240.

11. Chen CI, Paul H, Wang $\mathrm{T}$, et al. Long term follow up of a phase 2 trial of single agent lenalidomide in previously untreated patients with chronic lymphocytic leukaemia. Br J Haematol. 2014;165(5):731-733.

12. Badoux XC, Keating MJ, Wen S, et al. Lenalidomide as initial therapy of elderly patients with chronic lymphocytic leukemia. Blood. 2011;118(13):3489-3498.

13. Ferrajoli A, Lee B-N, Schlette EJ, et al. Lenalidomide induces complete and partial remissions in patients with relapsed and refractory chronic lymphocytic leukemia. Blood. 2008;111(11):5291-5297

14. James DF, Werner L, Brown JR, et al. Lenalidomide and rituximab for the initial treatment of patients with chronic lymphocytic leukemia: a multicenter clinical-translational study from the chronic lymphocytic leukemia research consortium. J Clin Oncol. 2014;32(19):2067-2073.

15. Thompson PA, Rozovski U, Keating MJ, et al. The addition of CD20 monoclonal antibodies to lenalidomide improves response rates and survival in relapsed/refractory patients with chronic lymphocytic leukaemia relative to lenalidomide monotherapy-the MD Anderson Cancer Center experience. Br J Haematol. 2015;171(2):281-284.

16. Ferrajoli A, Badoux XC, O'Brien S, et al. Combination therapy with lenalidomide and rituximab in patients with relapsed chronic lymphocytic leukemia (CLL). Blood. 2009;114(22):206-206.

17. Hallek M, Cheson BD, Catovsky D, et al. Guidelines for the diagnosis and treatment of chronic lymphocytic leukemia: a report from the International Workshop on Chronic Lymphocytic Leukemia updating the National Cancer Institute-Working Group 1996 guidelines. Blood. 2008;111 (12):5446-5456. 
18. Rawstron A, Böttcher S, Letestu $R$, et al. Improving efficiency and sensitivity: European Research Initiative in CLL (ERIC) update on the international harmonised approach for flow cytometric residual disease monitoring in CLL. Leukemia. 2013;27(1):142.

19. Cairo MS, Bishop M. Tumour lysis syndrome: new therapeutic strategies and classification. Br J Haematol. 2004;127(1):3-11.

20. Trotti A, Colevas AD, Setser A, et al. CTCAE v3. 0: development of a comprehensive grading system for the adverse effects of cancer treatment. Semin Radiat Oncol. 2003;13(3):176-181.

21. Hillmen P, Robak T, Janssens A, et al. Chlorambucil plus ofatumumab versus chlorambucil alone in previously untreated patients with chronic lymphocytic leukaemia (COMPLEMENT 1): a ran- domised, multicentre, open-label phase 3 trial. Lancet. 2015;385(9980):1873-1883.

22. Cheson B, Bennett J, Grever M, et al National Cancer Institute-sponsored Working Group guidelines for chronic lymphocytic leukemia: revised guidelines for diagnosis and treatment. Blood. 1996;87(12): 4990-4997.

23. O'Brien S, Furman RR, Coutre $S$, et al. Single-agent ibrutinib in treatment-naïve and relapsed/refractory chronic lymphocytic leukemia: a 5-year experience. Blood. 2018;131(17):1910-1919.

24. Badoux XC, Keating MJ, Wen S, et al. Phase II study of lenalidomide and rituximab as salvage therapy for patients with relapsed or refractory chronic lymphocytic leukemia. J Clin Oncol. 2013;31(5):584.

25. Fink AM, Bahlo J, Robrecht $S$, et al. Lenalidomide maintenance after first-line therapy for high-risk chronic lymphocytic leukaemia (CLLM1): final results from a randomised, double-blind, phase 3 study. Lancet Haematol. 2017;4(10):e475-e486.

26. Shanafelt TD, Ramsay AG, Zent CS, et al Long-term repair of T-cell synapse activity in a phase II trial of chemoimmunotherapy followed by lenalidomide consolidation in previously untreated chronic lymphocytic leukemia (CLL). Blood. 2013;121(20):41374141

27. Strati P, Keating MJ, Wierda WG, et al Lenalidomide induces long-lasting responses in elderly patients with chronic lymphocytic leukemia. Blood. 2013;122(5):734-737.

28. Fischer $\mathrm{K}, \mathrm{Al}$-Sawaf $\mathrm{O}$, Fink $\mathrm{A}-\mathrm{M}$, et al. Venetoclax and obinutuzumab in chronic lymphocytic leukemia. Blood. 2017;129 (19):2702-2705 